\title{
Transmuscular Quadratus Lumborum Block Reduces Length of Stay in Patients Receiving Total Hip Arthroplasty
}

\author{
Michael Stuart Green ${ }^{1,}{ }^{,}$, Christopher Ryan $\operatorname{Hoffman}^{1}$, Usama Iqbal ${ }^{1}$, Oluwafunke Olabisi Ives ${ }^{1}$ and \\ Benita Hurd ${ }^{1}$ \\ ${ }^{1}$ Department of Anesthesiology and Perioparative Medicine, Drexel University College of Medicine, Philadelphia, United States \\ "Corresponding author: Department of Anesthesiology and Perioparative Medicine, Drexel University College of Medicine, Philadelphia, United States. Tel: +1-8563085034, \\ E-mail:msg37@drexel.edu
}

Received 2018 June 06; Revised 2018 September 20; Accepted 2018 October 06.

\begin{abstract}
Background: Total hip arthroplasty is a common procedure being performed at an increasing rate in the United States. Recovering from this surgery to the extent that one can participate in criteria for discharge relies heavily on effective postoperative analgesia. Many regional anesthetic techniques are deployed in this realm. The recent utilization of quadratus lumborum (QL) blocks with success in other procedures warrants investigation in the hip arthroplasty population.

Methods: Twenty patients received general anesthesia for elective total hip arthroplasty. Ten cases included a preoperative ultrasound-guided transmuscular quadratus lumborum block with 30 cc $0.5 \%$ ropivacaine. Ten cases that lacked this regional procedure. The primary outcome was length of hospital stay. Secondary outcomes include total procedure time, intraoperative and postoperative fentanyl administration, and mean postoperative visual analog pain scores (VAS 1-10).

Results: Length of stay was shorter in patients receiving QL block (2.9 days) versus patients not receiving QL block (5.1 days) (P value 0.0146). Intra-operative use of fentanyl was lower in patients receiving QL block $(183.5 \mathrm{mcg})$ versus patients not receiving QL block (240 mcg) (P value 0.0376). PACU narcotic utilization, 24-hour VAS score, and length of operative procedure lacked statistical significance, though the study was not powered for these outcomes.

Conclusions: QL block employment in hip surgery produces significant reduction in length of stay and intraoperative fentanyl use. While quadratus lumborum blocks are rapidly becoming a popular option due to its quality and spread of analgesia, more adequately powered prospective research must be performed to appropriately elucidate significant trends.
\end{abstract}

Keywords: Analgesia, Analgesics, Peripheral Nervous System Agents, Anesthesia, Non-Narcotic, Anesthetics, Combined, Anesthetics, Local

\section{Background}

Hip pain is a common manifestation of hip pathology; the CDC reports that hip pain is 7th leading cause of chronic pain complaints in the United States (1). Osteoarthritis, a progressive disease, is one of the most common causes of hip pain. $14.3 \%$ of adults over 60 report significant hip pain on most days (2). Osteoarthritis was the diagnosis at the time of surgery for approximately $70 \%$ of patients undergoing hip arthroplasty (3). Osteoarthritis can be conservatively managed but therapy success and subsequent ability to work and perform is limited. Patients often find that surgical intervention is the only viable option for providing significant improvement in pain (3).

From 2000 to 2010 the number and rate of total hip replacements among patients 45 and over increased from
138.700 to 310.800 and 142.2 to 257.0 per 100.000 respectively (4). Total hip replacements are the second most predominant joint procedure performed in the U.S., comprising almost $40 \%$ of the total volume (3). The total inpatient cost of hip replacement surgery is estimated to range from $\$ 18.050$ to $\$ 43.700$. This fee includes the hip replacement surgery, anesthesia, and a 3-day hospital stay (5). Total hip replacements are considered to be cost effective over time due to benefits including increased ability to perform activities of daily life, improved quality of life for patient and caregivers, and decreased demand for community resources (6).

The quadratus lumborum (QL) nerve block, first introduced in 2007, was traditionally used for abdominal surgeries. Its application has broadened to include lower ex- 
tremity procedures (7). There are several approaches to the QL block: Lateral, posterior, anterior, and intramuscular, commonly termed QL1, QL2, QL3, and QL4 respectively. It is speculated that these differing approaches are responsible for the differing efficacy and spread observed between variants. Its spread has typically been reported ranging from T4-L1, however, in many observational reports, spread to lumbar nerves has been demonstrated (8). Carline et al. discovered that both lumbar and thoracic anterior nerve roots were consistently reached with the transmuscular variant of the QL block (9). Other reports found that QL blocks provided superior pain relief in comparison to femoral and fascia iliaca nerve blocks for postoperative analgesia (10). This technique was also associated with the added benefit of absent hip flexor and quadriceps weakness (11). Promising results have demonstrated effective postoperative analgesia in the T12-L2 dermatomes post hip arthroplasty on a case-by-case basis $(12,13)$.

In this study we review the length of stay and perioperative narcotic utilization in patients receiving transmuscular quadratus lumborum blocks for total hip arthroplasty. The primary outcome was length of hospital stay. Secondary outcomes measured include total procedure time, intraoperative and postoperative fentanyl administration, and mean postoperative visual analog pain scores (VAS 1 10). We discuss relevant anatomy, nerve block alternatives, and surgical considerations relevant to this topic.

\section{Methods}

Upon approval from our institutional review board, 20 patients ages 18 to 75 and ASA grade I, II or III were retrospectively reviewed in this study. No patient identifiers were recorded. All cases received general anesthesia for elective total hip arthroplasty. Ten cases included a preoperative ultrasound-guided transmuscular quadratus lumborum block while 10 cases that lacked this regional procedure. After obtaining consent, we placed the patient in lateral decubitus position. The field was sterilized with chlorhexidine and subsequently draped. A SonoSite ${ }^{\odot}$ M-Turbo (SonoSite Inc., Bothell, WA) ultrasound machine with a SonoSite linear transducer HFL50 (15 - 6 $\mathrm{MHz}$; Sonosite Inc.) identified relevant anatomy. The oblique and transversus abdominis muscles were identified anterior to the lateral border of the quadratus lumborum muscle. Subsequently the anterior border of the quadratus lumborum was the identified target. A Braun ${ }^{\odot}$
$21 \mathrm{Ga}$. X 4 inch insulated nerve block needle (BBraun, Melsungen, Germany) was directed via ultrasound guidance to the desired space. Thirty cc of $0.5 \%$ ropivacaine was administered. Patients subsequently underwent induction of general anesthesia and placement of an endotracheal tube with weight-based doses of intravenous fentanyl, lidocaine, propofol, and rocuronium. General anesthesia was maintained with sevoflurane or desflurane. Doses of bolus narcotic administered intraoperatively and in the PACU were retrospectively recorded. The study was not initially powered for secondary outcomes. Differences seen can lead to a larger scale study with powered sample sizes and a more comprehensive statistical analysis.

\section{Results}

In this equivalence study, individual data points were compared with two-sample $t$-test. Data analysis was performed with IBM SPSS (IBM Corp. Released 2016. IBM SPSS Statistics for Windows, Version 24.0. Armonk, NY: IBM Corp). P values of $<0.05$ were regarded as significant. Twenty patients completed the study, 10 in QL block group and 10 in non QL blockgroup (Table1). There was no statistical difference in the groups' age, ASA physical status, body mass index, and intraoperative time. Length of stay was shorter in patients receiving QL block (2.9 days) versus patients not receiving QL block (5.1 days) with statistical significance ( $P$ value 0.0146). Intra-operative use of fentanyl was lower in patients receiving QL block $(183.5 \mathrm{mcg})$ versus patients not receiving QL block $(240 \mathrm{mcg})$ with statistical significance (P value 0.0376). Other parameters including PACU narcotic utilization, 24-hour VAS score lacked statistical significance, though the study was not powered for these outcomes.

Another observed finding is three unblocked patients were deemed appropriate for patient-controlled analgesia (PCA). While they were not heavily utilized, their clinical assessment appeared to dictate that a PCA be set up. This may have contributed to patient perception regarding participating in physical therapy.

\section{Discussion}

Preliminary case reports demonstrate improved analgesia among those receiving QL blocks for hip arthroplasty. Data presented here supports the argument that QL block success leads to further improvements in clinical outcome, namely length of stay. We attribute this improved outcome 


\begin{tabular}{|c|c|c|c|}
\hline Parameters & With QL Block ${ }^{a}$ & Without QL Block $^{\mathbf{a}}$ & PValue \\
\hline Procedure time, $\min$ & $186.4(28.9)$ & $175.1(47.7)$ & 0.5338 \\
\hline Intra-op fentanyl, mcg & $183.5(62.4)$ & $240(51.6)$ & 0.0376 \\
\hline PACU fentanyl, mcg & $45(59.8)$ & $25(42.4)$ & 0.4002 \\
\hline Length of stay, days & $2.9(1.3)$ & $5.1(2.2)$ & 0.0146 \\
\hline
\end{tabular}

${ }^{\mathrm{a}}$ Values are expressed as mean (SD).

to be secondary to improved analgesia and subsequent participation in physical therapy. We aimed to demonstrate a lack of difference in patient anatomy in identifying no statistical difference in age, BMI, and ASA physical status. Block difficulty was not shown to be an issue as evidence of increased operative time was not found.

It is noted that 24-hour VAS pain scores and narcotic utilization was not statistically significant in this report. It is plausible that given a larger sample size, these measures may follow the same trend as length of stay and would be consistent with case reporting (11-13). We did not control for when VAS scores were ascertained, and thus scores may have been assessed at varying times. It would be reasonable that the comforted patient at rest may report differently than those recently returning from physical therapy sessions, for example. Lacking controlled timing of this postoperative measurement may introduce bias. The same could be attributed to administering breakthrough medication at rest versus after physical therapy sessions. When expanding this investigation to a larger population, these measures should be addressed. We also did not investigate the extent of motor blockade present or absent during the recovery process. Prior case reporting has included absent motor blockade that facilitates physical therapy while reducing discomfort (11). We pose the argument that increased physical therapy and decreased length of stay demonstrates this trend but larger scale studies would benefit from including sensorimotor examination as a measured outcome. Perioperative narcotic administration was retrospectively recorded. A large-scale prospective trial with metered narcotic intervention and routine postoperative pain regimen protocol via patientcontrolled analgesia pump is recommended to eliminate provider-to-provider variety in assessment and administration.

Block selection may be dependent on surgical approach (anterior, posterior, lateral). The anterior approach is becoming more popular due its muscle sparing properties, which has led to reduced invasiveness. This technique has been associated with improved recovery and rehabilitation times and subsequently shorter hospital stays. Significant VAS score reduction and narcotic consumption are also reported in the anterior approach (14). Varied approaches lead to different nerve distributions requiring coverage and can change optimal block selection. Surgeons at our facility utilize the posterior approach. Alternatives interventions including neuraxial anesthesia, femoral nerve block, fascia iliaca nerve block, lumbar plexus block, and erector spinae block may provide more optimal coverage depending on surgical approach. Larger scale studies comparing multiple blocks may require accounting for varying regions affected by surgical manipulation.

It is important to understand the criteria for discharge dictated by the surgical service as anesthetic techniques may facilitate said goals. Criteria for discharge includes maintaining acceptable pain control, activities of daily living (bedding, eating, toileting), ambulation with assistive devices, and relevant physical therapy exercises. Poor pain management during the perioperative course is associated with delayed ambulation, pulmonary complications, and delayed transition to lower levels of care (15). Narcoticsparing peripheral nerve blockade resulting in decreased cost and improved overall patient satisfaction. Improving nerve block selection, deployment, and efficacy is therefore a joint anesthetic and surgical endeavor.

\subsection{Conclusion}

Hip disorders are one of the most common causes of chronic pain in the United States and both the physical and financial burdens of these processes are daunting. Hip joint surgery must be performed with the appropriate anesthetic considerations in mind to ensure patient comfort, appropriate utilization of resources, and improved 
postoperative course metrics (length of stay, narcotic usage, postoperative complications, etc). There are several peripheral nerve block techniques that can be used for hip surgery, each with their own respective advantages and disadvantages. Many recent observational studies have found that QL blocks can be extensively used for a multitude of different procedures. Its employment in hip surgery has been successful and is associated with several advantages. This study found significant reduction in length of stay and intraoperative fentanyl use. Many factors may have contributed to the finding of decreased LOS for the QL block group. Although improved VAS scores did not show statistical significance in this study, it is clear that obstacles to physical therapy like pain are the main stay of hurdles prolonging hospital stay for surgical orthopedic patients. It is logical to posit that a decreased LOS almost necessitates improved, or at the very least adequate, participation in physical therapy and improved overall pain control. These findings, if founded, have paramount potential clinical significance for everyday practice and treatment of surgical orthopedic patients. The main limitation of this study is the sample size powered for secondary outcomes. To evaluate the significance and implication of these claims, further studies would be necessary to explore beyond the scope of the current study.

\section{Footnote}

\section{Ethical Considerations: IRB 1710005726.}

\section{References}

1. Institute of Medicine of the National Academies Report. Relieving pain in America: A blueprint for transforming prevention, care, education, and research. Washington DC: The National Academies Press; 2011. 62 p.

2. Wilson JJ, Furukawa M. Evaluation of the patient with hip pain. Am Fam Physician. 2014;89(1):27-34. [PubMed: 24444505].
3. American Joint Replacement Registry Report. Third AJRR annual report on hip and knee arthroplasty data: Annual report. 2016.14 p.

4. Wolford M, Palso K, Bercoviz A. Hospitalization for total hip replacement among inpatients aged 45 and Over: United States, 2000-2010. Centers for Disease Control and Prevention, National Center for Health Statistics Brief. 2015.

5. Healthcare Blue Book. Total hip replacement. Health care blue book.2018, [cited March 21]. Available from: healthcarebluebook.com.

6. Wilcock GK. Benefits of total hip replacement to older patients and the community. Br Med J. 1978;2(6129):37-9. [PubMed: 678799] [PubMed Central: PMC1605736].

7. Akerman M, Pejčić N, Veličković I. A review of the quadratus lumborum block and ERAS. Front Med (Lausanne). 2018;26(5):44. doi: 10.3389/fmed.2018.00044. [PubMed: 29536008]. [PubMed Central: PMC5834926].

8. Ueshima $\mathrm{H}$, Otake $\mathrm{H}$, Lin JA. Ultrasound-guided quadratus lumborum block: An updated review of anatomy and techniques. Biomed Res Int. 2017;2017. doi: 10.1155/2017/2752876. [PubMed: 28154824]. [PubMed Central: PMC5244003].

9. Carline L, McLeod GA, Lamb C. A cadaver study comparing spread of dye and nerve involvement after three different quadratus lumborum blocks. Br J Anaesth. 2016;117(3):387-94. doi: 10.1093/bja/aew224. [PubMed: 27543534].

10. La Colla L, Ben-David B, Merman R. Quadratus lumborum block as an alternative to lumbar plexus block for hip surgery: a report of 2 cases. A A Pract. 2017;8(1):4-6. doi: 10.1213/XAA.0000000000000406. [PubMed: 28036319].

11. Hockett MM, Hembrador S, Lee A. Continuous quadratus lumborum block for postoperative pain in total hip arthroplasty: A case report. A A Case Rep. 2016;7(6):129-31. doi: 10.1213/XAA.0000000000000363. [PubMed: 27513972].

12. Ueshima $\mathrm{H}$, Yoshiyama $\mathrm{S}$, Otake $\mathrm{H}$. The ultrasound-guided continuous transmuscular quadratus lumborum block is an effective analgesia for total hip arthroplasty. J Clin Anesth. 2016;31:35. doi: 10.1016/j.jclinane.2015.12.033. [PubMed: 27185672].

13. Johnston DF, Sondekoppam RV. Continuous quadratus lumborum block analgesia for total hip arthroplasty revision. J Clin Anesth. 2016;35:235-7. doi: 10.1016/j.jclinane.2016.08.002. [PubMed: 27871532].

14. Petis S, Howard JL, Lanting BL, Vasarhelyi EM. Surgical approach in primary total hip arthroplasty: Anatomy, technique and clinical outcomes. Can J Surg. 2015;58(2):128-39. [PubMed: 25799249]. [PubMed Central: PMC4373995].

15. Abou-Setta AM, Beaupre LA, Jones CA. Pain management interventions for hip fracture [Internet]. Rockville (MD): Agency for Healthcare Research and Quality (US); 2011. Comparative effectiveness reviews. 